\title{
NONLINEAR AUTOREgRESSIVE NETWORK WITH The Use Of A Moving Average Method For FORECASTING TYPHOON TRACKS
}

\author{
Tienfuan Kerh and Shin-Hung Wu \\ Department of Civil Engineering \\ National Pingtung University of Science and Technology, Pingtung, Taiwan.
}

\begin{abstract}
Forecasting of a typhoon moving path may help to evaluate the potential negative impacts in the neighbourhood areas along the moving path. This study proposed a work of using both static and dynamic neural network models to link a time series of typhoon track parameters including longitude and latitude of the typhoon central location, cyclonic radius, central wind speed, and typhoon moving speed. Based on the historical records of 100 typhoons, the performances of neural network models are evaluated from the indices of a correlation coefficient and a mean square error. The dynamic model or the so-called nonlinear autoregressive network with the use of a moving average method proved to forecast the ten types of typhoon moving path more effectively in Taiwan region. The new and simply approach developed in this study for solving studied typhoon cases may be applicable to other areas of interest worldwide..
\end{abstract}

\section{KEYWORDS}

Typhoon moving path, static model, dynamic model, moving average method, evaluation index.

\section{INTRODUCTION}

Typhoon is one of natural phenomenon that generates a very powerful wind shear and its induced effects such as flooding and land sliding may cause tremendous property damages and loss of human lives, depended on the strength and the moving path of the typhoon. The genesis of typhoons is mostly located in the region of western North Pacific Ocean according to past records. Following the development process of typhoons in this region, there are about 26 typhoons in average occurred yearly, where about three to four typhoons raided the island of Taiwan distributed mostly between July to September [1]. Although the relevant government agency of Taiwan has established a warning system, the accuracy for forecasting the moving path of typhoons still needs to be improved for reducing the negative impacts. As the typhoons may have an influence on several countries, so the typhoon related topics keep studying by many academic researchers in the international community.

Basically, there are three types of models including statistical, dynamical, and combined statistical-dynamic models, for forecasting typhoon track, intensity, storm surge, rainfall, and the area threatened. No matter which model, it relies on the use of meteorological data that may be obtained from satellites, land-based radar, and reconnaissance aircrafts [2-3]. For more specific, the parameters to position the typhoon center may include surface pressure, wind circulation center, and the cloud system center. To reduce the forecasting errors from the above mentioned models, the theory of probability has been adopted to express the uncertainty in typhoon motion forecasts through the construction of probability ellipses [4-6]. Other forecasting models of typhoon path may also be found in many of previous literatures (e.g. [7-9]). No surprisingly, these 
typhoon forecasting methods all involve a very complicated mathematical model and may require a heavy computational cost to obtain reliable results.

The recently developed artificial intelligence also plays an important role for forecasting typhoon related research topics. For instances, the artificial neural network is applied for forecasting precipitation during typhoon periods by taking observed typhoon characteristics and ground weather data as the basis [10]. Wave peak or storm surge derivation due to typhoon at a point of interest and extended to other points could be predicted by using neural network approach with different analysis functions [11-12]. Although the applications of using artificial intelligence for predicting typhoon moving track based on meteorological fields are also available, these researches tended to a very complicated manner [13-14], and that may open an opportunity to study this interesting topic in an easier way.

This study focuses on the development of static and dynamic neural network models for forecasting typhoon moving path by taking a series of time steps of typhoon data recorded previously. The parameters from typhoon historical records including the coordinates (longitude, latitude) of typhoon location, cyclonic radius, wind speed at central point, and typhoon moving speed are used for training, adapting, and verification the models. From statistical analysis, there exist ten types of typhoon moving paths occurred on the Taiwan region, and there are 100 typhoon cases recorded previously in 1972-2012 are taken to develop the models. The performances of neural network models are evaluated from a correlation coefficient and a mean square error, and the forecasting result is improved with the use of moving average method. In addition, the recently occurred typhoons in 2013-2016 are forecasted without through training process to further validate the preferred models in the present study. Without considering the complicated weather condition or using meteorological data, the approach proposed in this study may provide a simple and cost inexpensive way for forecasting typhoon moving tracks.

\section{TYPHOON INFORMATION AND DATA SOURCE}

In general cases, the generation of typhoons in western North Pacific Ocean is mainly guided by circulation of subtropical high atmospheric pressure, and most of typhoons track has a tendency to move west direction. But when typhoons move to nearby of Taiwan and Philippine areas, the moving paths become changeable due to the rim of subtropical high atmospheric pressure. Some of typhoons keep moving toward west direction, some of them transfer moving toward northeast direction, and some of them keep staying at the same location. Based on the statistical analysis of typhoon moving path records from Central Weather Bureau (CWB) of Taiwan for the recent hundred years, there are ten types of typhoon moving path can be drawn and shown in Figure 1 (left). For the ten types of typhoons as displayed in Figure 2 (right), the occurrences of each type are: Type $1(13 \%)$, Type 2 (11\%), Type 3 (11\%), Type 4 (10\%), Type $5(19 \%)$, Type $6(16 \%)$, Type 7 (7\%), Type 8 (4\%), Type 9 (7\%), and Type $10(2 \%)$, respectively.

In accordance with the historical typhoon data base from CWB, this study collects five typhoon related parameters including altitude, latitude, cyclonic radius, central wind speed, and moving speed, occurred during the recent four decades [15]. As some of typhoon event cases in early years were recorded by hand writing, which may not well clarified and recognized, and that results in a tendency of incompleteness. Hence, this study tried to pick up typhoon data sources as newer as possible for completeness and for analysis. Additionally for the sake of convenience, the data sets of 100 typhoon events in accordance with the occurred percentages of the ten typhoon types are chosen for numerical computation by neural network approach.

The original data sets show that some of typhoon moving paths are recorded for every 3 or 6 hours during at sea, whereas every 1 or 3 hours during at land. In order to unify the time interval 
of typhoon records, this study uses 1 hour time interval for each typhoon path record, and a time series of data is applied in neural network analysis. To prevent extreme values and to increase convergent speed in neural computing, the data sets of typhoon moving path need to be normalized by using the following linear transformation equation:

$$
Y=\left(X-X_{\min }\right) /\left(X_{\max }-X_{\min }\right)
$$

where $X$ denotes the value before normalization; $Y$ represents the value after normalization; $X_{\min }$ and $X_{\max }$ are the minimum and maximum values in original numerical sequences, respectively.
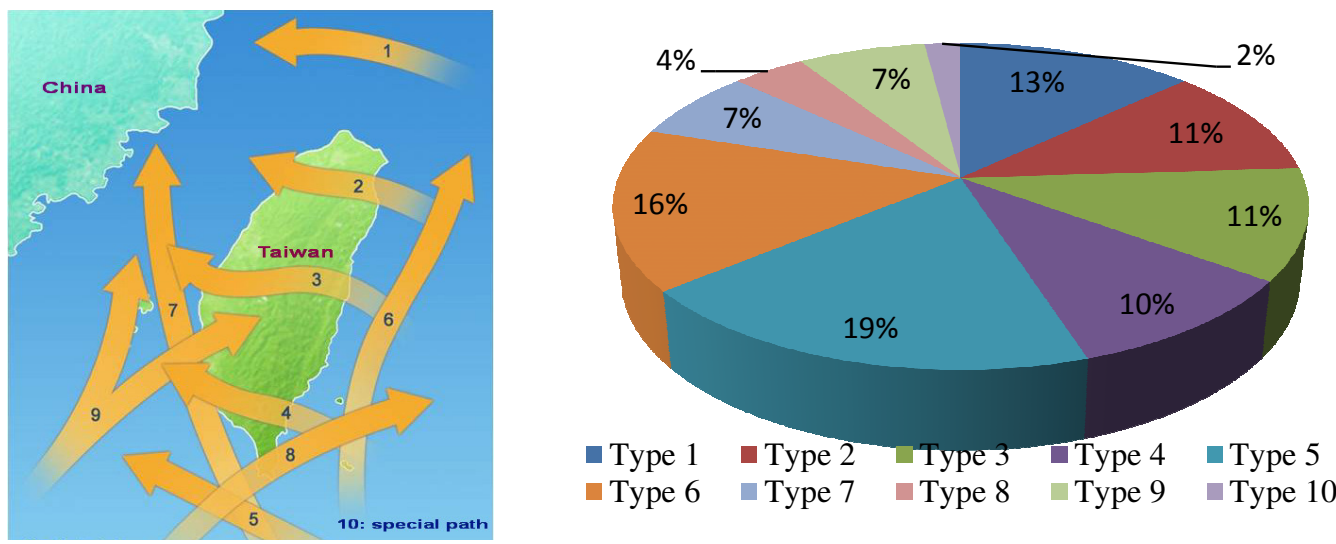

Figure 1. Statistical ten types of typhoon path and the occurrences in Taiwan region [15].

\section{Static and Dynamic Neural Network Models}

\subsection{Structure of Neural Network Models}

Due to the advantages of simplicity and effectiveness, the artificial neural network has been widely used in various applications recently. The basic element of a multi-layered neural network model constitute a series of neurons, where a set of weights, an adder, and a transfer function are performed to link neurons in different layers. The following mathematical equation can be written to describe the principle of a neural network model:

$$
y_{j}=F\left(\sum_{i=1}^{n} w_{i j} x_{i}-b_{j}\right)
$$

where $y_{i}$ is the output of neuron $j, w_{i j}$ represents the weight from neuron $i$ to neuron $j, x_{i}$ is the input signal generated for neuron $i$, and $b_{j}$ is the bias term associated with neuron $j$. The activation function $F$ is used to constraint the output values to be between $[0,1]$ or $[-1,1]$ for the input values ranging from negative to positive infinity. This nonlinear transfer function can make the operating process continuous and differentiable.

The artificial neural network may be in general classified into two types: static neural network model and dynamic neural network model; depends on the treatment of time factor $(t)$ in the analysis. For the static neural network model, the corresponding relation between input and output will not be varied with the time factor, as this factor is implicit in the input parameters. Whether to consider the time factor or not, the use of static neural network can be found widely in 
various applications (e.g. [16-20]). Relatively, the time factor is explicit represented in the dynamic neural network model, by using feedback loop to cause time delays. Further to mention that the dynamic neural network is not only treat nonlinear multivariate behaviour, but also include learning of time-dependent behaviour such as various transient phenomena and delay effects [21-25]. Nevertheless, both neural network models can be applied to analyse the present research topic in typhoon path prediction problem.

The structures of static and dynamic neural network models may be shown in Figure 2 (left) and Figure 2 (right) respectively [26]. For the structure of static neural network, it denotes that there are $\mathrm{n}$ parameters in input $\mathrm{x}$, and there are $\mathrm{m}$ time factors in each of input parameter. In addition, the sequential parameters $x_{1}(t), x_{1}(t-1), x_{1}(t-2)$ for example, are taken as the inputs in the time delay backpropagation neural networks. For the dynamic neural network structure, there are $\mathrm{m}$ parameters in input $x, n$ time delays in $y(t), k$ parameters in output $z(t+1)$, and the symbols $v$ and $w$ represent weight and bias values either in input or feedback layer. From these figures, it is easier to understand the operating process of the two neural network models. Regarding the calculation processes of the two models is described in the next subsection. In the present study, the software package MATLAB is taken to perform calculation of the two models [27-28], where the backpropagation neural network (BP, a feedforward static model) and the nonlinear autoregressive network (NARX, a recurrent dynamic model), are chosen for analysing a series of typhoon data sets.
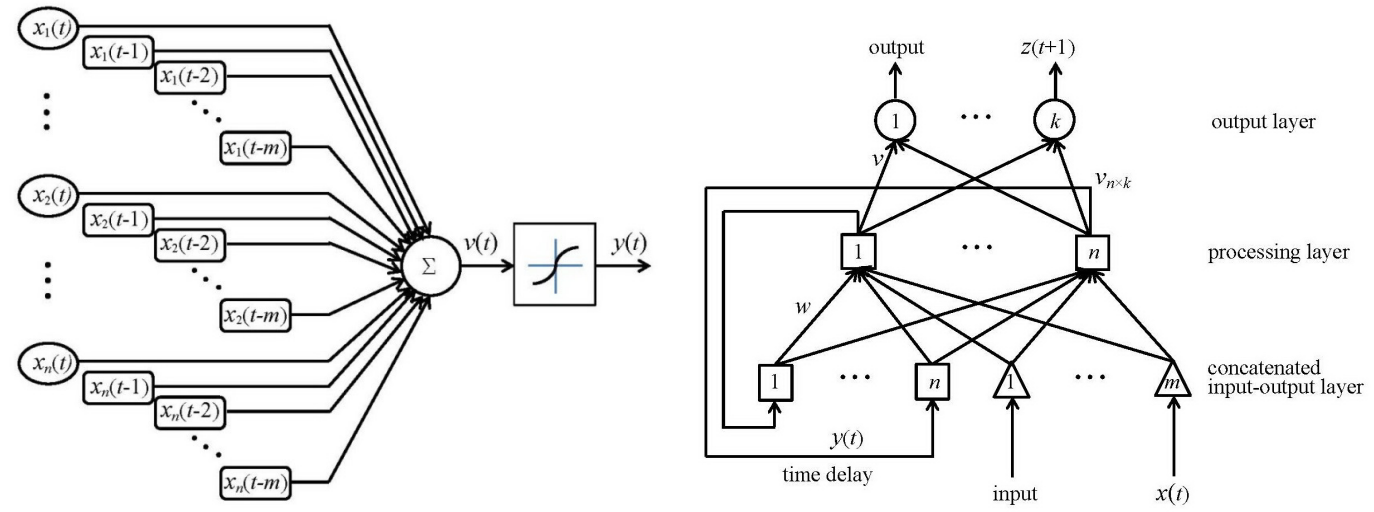

Figure 2. Structure of the static (left) and dynamic neural network model (right).

\subsection{Algorithm of Neural Network Models}

The basic and extended theoretical backgrounds for the neural network approach can be found in many relevant references [29-30]. For simplification, the operational steps of a multi-layered back-propagation neural network approach can be described as follows: (1) Calculate output value in the hidden layer; (2) Calculate output value in the output layer; (3) Calculate gap amount of neuron in the output layer; (4) Calculate gap amount of neuron in the hidden layer; (5) Calculate corrections of weight matrix and bias vector in the output layer; (6) Calculate corrections of weight matrix and bias vector in the hidden layer. Note that the steps (1) and (2) are forward calculations, and the steps from (3) to (6) are reverse adjustments for weight and bias term. The detailed mathematical equations are not shown in here as they can be easily found in previous indicated references. Briefly to say is that the neural network training process can be performed by repeating the six steps until the error tolerance is achieved. 
For a dynamic neural network model, the often adopted real-time recurrent learning algorithm is depicted more in detail here based on the cited references [31-32]. Initially, by defining that $I$ represents a set of input units, $I=\left\{x_{k}(t), 0<k<m\right\}$, and $U$ is a set of other units, $U=\left\{y_{k}(t), 0<k<n\right\}$, which can be hidden or output units. Then, indexing that $z_{k}$ is an arbitrary unit in the network as that

$$
z_{k}(t)= \begin{cases}x_{k}(t) & \text { if } k \in I \\ y_{k}(t) & \text { if } k \in U\end{cases}
$$

By computing weighted sum of the inputs for a dynamic system, the nonlinear function can be written as follows:

$$
y_{k}(t+1)=f_{k}\left[\operatorname{net}_{k}(t)\right]=f_{k}\left[\sum_{l \in U \cup I} w_{k l} z_{i}(t)\right]
$$

where $w_{k l}$ is the weight to neuron $k$ from neuron $l$. Now by assuming that $d_{k}(t)$ is the target value of the $k$ neuron at time step $t$, and $e_{k}(t)$ represents an error for the neuron $k$ at time step $t$. Then, the transient error function $E(t)$ can be written as the following equation:

$$
E(t)=\frac{1}{2} \sum_{k \in U} e_{k}^{2}(t)=\frac{1}{2} \sum_{k \in U}\left[d_{k}(t)-y_{k}(t)\right]^{2}
$$

By applying the steepest gradient descent method, and a time series is presented to the network, the weight changes $\Delta w_{i j}$ from an accumulation of the gradient values may be written as:

$$
\Delta w_{i j}(t)=-\eta \frac{\partial E(t)}{\partial w_{i j}}
$$

where $\eta$ is the parameter of learning rate. After the network has been calculated for the whole time series, the use of chain rule at each time step $t$ gives:

$$
-\frac{\partial E(t)}{\partial w_{i j}}=\frac{\partial E(t)}{\partial y_{k}(t)} \frac{\partial y_{k}(t)}{\partial w_{i j}}=\sum_{k \in U} e_{k}(t) \frac{\partial y_{k}(t)}{\partial w_{i j}}
$$

Since the error $e_{k}(t)$ at all times can be calculated from the difference between targets and outputs, the remaining derivation term can be calculated from equation (4) as that:

$$
\frac{\partial y_{k}(t+1)}{\partial w_{i j}}=f_{k}^{\prime}\left[\operatorname{net}_{k}(t)\right]\left[\sum_{l \in U \cup I} w_{k l} \frac{\partial z_{l}(t)}{\partial w_{i j}}+\delta_{i k} z_{j}(t)\right]
$$

where $\delta_{i k}$ is the Kronecker delta. Since $\frac{\partial z_{l}(t)}{\partial w_{i j}}=0$ for $l \in I$, so equation (8) may become:

$$
\frac{\partial y_{k}(t+1)}{\partial w_{i j}}=f_{k}^{\prime}\left[n e t_{k}(t)\right]\left[\sum_{l \in U} w_{k l} \frac{\partial y_{l}(t)}{\partial w_{i j}}+\delta_{i k} z_{j}(t)\right]
$$

The above equation is a recursive equation, so it is possible to compute the value for time step $t+1$, if the value of previous time step $t$ is known. 
By setting the initial condition at $t=t_{0}$ as $\frac{\partial y_{k}\left(t_{0}\right)}{\partial w_{i j}}=0$, for all $k \in U, i \in U$ and $j \in U \cup I$; and defining the variable $p_{i j}^{k}(t)=\frac{\partial y_{k}(t)}{\partial w_{i j}}$ for every time step $t$ and for all appropriate $i, \mathrm{j}$, and $k$. With the initial condition: $p_{i j}^{k}\left(t_{0}\right)=0$, the equation for each time step $t$ is:

$$
p_{i j}^{k}(t+1)=f_{k}^{\prime}\left[\operatorname{net}_{k}(t)\right]\left[\sum_{l \in U} w_{k l} p_{i j}^{l}(t)+\delta_{i k} z_{j}(t)\right]
$$

From the differences between targets and actual outputs, the weight changes $\Delta w_{i j}(t)$ and the overall correction $\Delta w_{i j}$ can be obtained as:

$$
\Delta w_{i j}=\sum_{t=t_{0}+1}^{t_{1}} \Delta w_{i j}(t)=\sum_{t=t_{0}+1}^{t_{1}} \eta \sum_{k \in U} e_{k}(t) p_{i j}^{k}(t)
$$

The above derivation and description may complete the calculation algorithm of a dynamic neural network model, and can be used for forecasting typhoon moving track in this study.

\section{Performance and Forecasting of Typhoon Moving Track}

\subsection{Comparison of Neural Network Models}

To develop a neural network model, the normalized typhoon record parameters including longitude $(l)$, latitude $(a)$, cyclonic radius $(r)$, wind speed at central point $(w)$, and moving speed $(m)$, are initially inputted with different combination of these parameters in the input layer. Then, by using different neurons in the hidden layer in the model, the parameters of altitude and latitude can be obtained in the output layer, for three neural network calculation stages (train, validation, and test). For having a sufficient accuracy, and for preventing over training and reducing training time, three convergent criteria are set as: (1) 1000 learning cycles, (2) mean square error (mse) less than $10^{-5}$, and (3) the least mse of 6 validation checks. The execution program will be terminated as one of these criteria is reached.

The definition of mean square error is:

$$
\mathrm{mse}=\frac{1}{n} \sum_{t=1}^{N}\left(d_{i}-y_{i}\right)^{2}
$$

where $d_{i}$ is the recorded value; $y_{i}$ is the calculated value; and $N$ denotes the data number in the analysis. Sometimes, the root mean square error (rmse) may also be used to express the accuracy of calculation. In addition, by calculating the averaged value of record data $\bar{d}$ and calculated results $\bar{y}$, the coefficient of correlation $(R)$ can be defined as:

$$
R=\frac{\sum_{t=1}^{N}\left(d_{i}-\bar{d}\right)\left(y_{i}-\bar{y}\right)}{\sum_{t=1}^{N}\left(d_{i}-\bar{d}\right)^{2} \sum_{t=1}^{N}\left(y_{i}-\bar{y}\right)^{2}}
$$


As the coefficient of correlation may exhibit a negative value, the square value $\left(R^{2}\right)$ is frequently used to evaluate the relationship between records and neural network calculations. In general case, the higher the $R^{2}$ value, the better the prediction result.

Now by taking typhoon Type 1 as an example case, shown in Table 1 is the comparison result of back-propagation neural network models with different input parameters and neurons in the hidden layer. Note that one hidden layer is sufficient to analysis in this typhoon path problem studied. It can be found that the model $\mathrm{I}_{l a w} \mathrm{H}_{3} \mathrm{O}_{l a}$ has a relatively better performance as its calculated $\mathrm{R}^{2}$ value reaches up to 0.9977 . That is, the static model of input parameters (altitude, latitude, and wind speed at central point), with three neurons in the hidden layer, can have a highly reliability to describe typhoon Type 1 . For the comparison result of dynamic neural network models, Table 2 shows that a model of similar input parameters as the static model, with two neurons in the hidden layer $\mathrm{I}_{l a w} \mathrm{H}_{2} \mathrm{O}_{l a}$, can obtain a relatively better value of $R^{2}=0.9970$, which may imply that the dynamic model can also be used to solve this type of typhoon path forecasting problem efficiently.

As displayed in Figure 3 (a) and (b) are the plot comparison of normalized coordinates (xaltitude, y-latitude) of typhoon path Type 1 for the two neural network models. It can be seen that both static model and dynamic model are in good agreements with recorded data. Similarly to the above analysis, the relatively better models can be obtained for the other nine typhoon types. Table 3 shows the performance of each typhoon type, and it can be found that both models exhibit a very good performance as all correlation coefficients fall in very high values. The averaged value of the ten typhoon types for the BP model is 0.9875 , and it is 0.9909 for the NARX model, so the dynamic model is slightly better than that of the static model here in this typhoon type case. From the table, it also can be found that each of the five input parameters may play an important role in different typhoon types. Therefore, it will not skip any one from the five input parameters in the following further analysis of typhoon path problems.

Table 1. Comparison result of different static neural network models for typhoon Type 1.

\begin{tabular}{|c|c|c|c|c|c|c|c|}
\hline $\mathrm{BP}$ & $R^{2}$ & $\mathrm{BP}$ & $R^{2}$ & $\mathrm{BP}$ & $R^{2}$ & $\mathrm{BP}$ & $R^{2}$ \\
\hline$I_{l a} H_{2} O_{l a}$ & 0.9972 & $I_{l a w} H_{5} O_{l a}$ & 0.8882 & $I_{\text {larw }} H_{6} O_{l a}$ & 0.9848 & $I_{\text {lawm }} H_{5} O_{l a}$ & 0.8552 \\
\hline$I_{l a} H_{3} O_{l a}$ & 0.9833 & $I_{l a w} H_{6} O_{l a}$ & 0.9455 & $I_{\text {larw }} H_{7} O_{l a}$ & 0.2568 & $I_{\text {lawm }} H_{6} O_{l a}$ & 0.9910 \\
\hline$I_{l a} H_{4} O_{l a}$ & 0.9859 & $I_{l a m} H_{3} O_{l a}$ & 0.9956 & $I_{\text {larm }} H_{3} O_{l a}$ & 0.8098 & $I_{\text {lawm }} H_{7} O_{l a}$ & 0.9718 \\
\hline$I_{l a r} H_{3} O_{l a}$ & 0.5375 & $I_{l a m} H_{4} O_{l a}$ & 0.9884 & $I_{\text {larm }} H_{4} O_{l a}$ & 0.4977 & $I_{\text {larwm }} H_{4} O_{l a}$ & 0.8494 \\
\hline$I_{l a r} H_{4} O_{l a}$ & 0.2805 & $I_{l a m} H_{5} O_{l a}$ & 0.9743 & $I_{\text {larm }} H_{5} O_{l a}$ & 0.6699 & $I_{\text {larwm }} H_{5} O_{l a}$ & 0.5803 \\
\hline$I_{l a r} H_{5} O_{l a}$ & 0.9784 & $I_{\text {lam }} H_{6} O_{l a}$ & 0.9912 & $I_{\text {larm }} H_{6} O_{l a}$ & 0.3917 & $I_{\text {larwm }} H_{6} O_{l a}$ & 0.5777 \\
\hline$I_{l a r} H_{6} O_{l a}$ & 0.9769 & $I_{l a r w} H_{3} O_{l a}$ & 0.9364 & $I_{\text {larm }} H_{7} O_{l a}$ & 0.9846 & $I_{\text {larwm }} H_{7} O_{l a}$ & 0.9430 \\
\hline$I_{l a w} H_{3} O_{l a}$ & $\mathbf{0 . 9 9 7 7}$ & $I_{l a r w} H_{4} O_{l a}$ & 0.2492 & $I_{l a w m} H_{3} O_{l a}$ & 0.9960 & $I_{\text {larwm }} H_{8} O_{l a}$ & 0.9729 \\
\hline$I_{\text {law }} H_{4} O_{l a}$ & 0.9968 & $I_{\text {larw }} H_{5} O_{l a}$ & 0.9592 & $I_{\text {lawm }} H_{4} O_{l a}$ & 0.9248 & $I_{\text {larwm }} H_{9} O_{l a}$ & 0.9815 \\
\hline
\end{tabular}


International Journal of Artificial Intelligence and Applications (IJAIA), Vol.8, No.6, November 2017

Table 2. Comparison result of different dynamic neural network models for typhoon Type 1.

\begin{tabular}{|c|c|c|c|c|c|c|c|}
\hline NARX & $R^{2}$ & NARX & $R^{2}$ & NARX & $R^{2}$ & NARX & $R^{2}$ \\
\hline$I_{l a} H_{l} O_{l a}$ & 0.9896 & $\boldsymbol{I}_{l a w} \mathrm{H}_{2} \mathrm{O}_{l a}$ & 0.9970 & $\mathrm{I}_{\text {larw }} \mathrm{H}_{2} \mathrm{O}_{l a}$ & 0.9920 & $\mathrm{I}_{\text {lawm }} \mathrm{H}_{2} \mathrm{O}_{\text {la }}$ & 0.9857 \\
\hline $\mathrm{I}_{l a} \mathrm{H}_{2} \mathrm{O}_{l a}$ & 0.9871 & $I_{l a w} H_{3} O_{l a}$ & 0.9946 & $\mathrm{I}_{\text {larw }} \mathrm{H}_{3} \mathrm{O}_{l a}$ & 0.9763 & $\mathrm{I}_{\text {lawm }} \mathrm{H}_{3} \mathrm{O}_{\text {la }}$ & 0.9378 \\
\hline $\mathrm{I}_{l a} \mathrm{H}_{3} \mathrm{O}_{l a}$ & 0.9745 & $I_{l a w} H_{4} O_{l a}$ & 0.9963 & $\mathrm{I}_{\text {larw }} \mathrm{H}_{4} \mathrm{O}_{l a}$ & 0.9774 & $\mathrm{I}_{\text {lawm }} \mathrm{H}_{4} \mathrm{O}_{\text {la }}$ & 0.9710 \\
\hline $\mathrm{I}_{\text {lar }} \mathrm{H}_{2} \mathrm{O}_{l a}$ & 0.9887 & $\mathrm{I}_{\text {lam }} \mathrm{H}_{2} \mathrm{O}_{l a}$ & 0.9836 & $\mathrm{I}_{\text {larm }} \mathrm{H}_{2} \mathrm{O}_{\text {la }}$ & 0.8415 & $\mathrm{I}_{\text {larwm }} \mathrm{H}_{2} \mathrm{O}_{l a}$ & 0.9917 \\
\hline $\mathrm{I}_{l a r} \mathrm{H}_{3} \mathrm{O}_{l a}$ & 0.9566 & $\mathrm{I}_{\text {lam }} \mathrm{H}_{3} \mathrm{O}_{l a}$ & 0.9881 & $\mathrm{I}_{\text {larm }} \mathrm{H}_{3} \mathrm{O}_{\text {la }}$ & 0.9821 & $\mathrm{I}_{\text {larwm }} \mathrm{H}_{3} \mathrm{O}_{\text {la }}$ & 0.9828 \\
\hline $\mathrm{I}_{l a r} \mathrm{H}_{4} \mathrm{O}_{l a}$ & 0.9905 & $I_{l a m} H_{4} O_{l a}$ & 0.9430 & $\mathrm{I}_{\text {larm }} \mathrm{H}_{4} \mathrm{O}_{\text {la }}$ & 0.8689 & $I_{\text {larwm }} H_{4} O_{l a}$ & 0.9782 \\
\hline
\end{tabular}

(a)

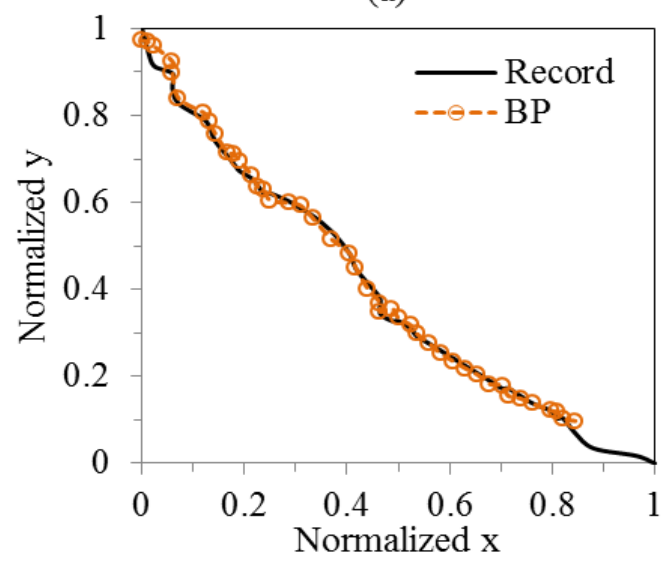

(b)

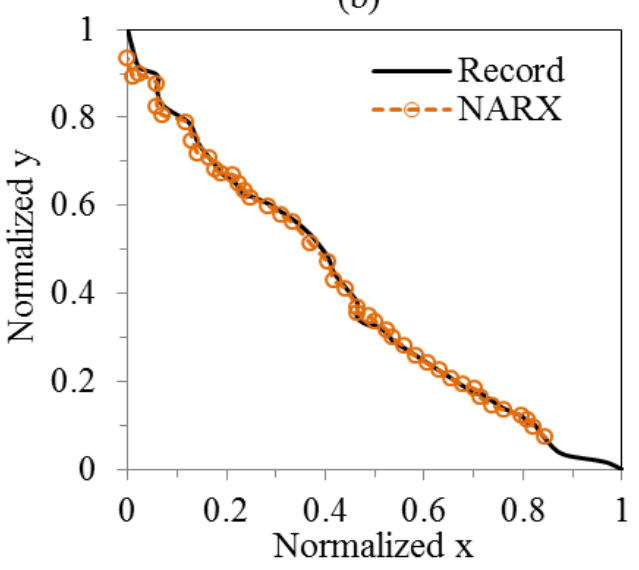

Figure 3. Plot comparison of normalized coordinates for typhoon path Type 1. (a) static model, (b) dynamic model

Table 3. The relatively better neural network models for the ten typhoon types.

\begin{tabular}{|c|c|c|c|c|}
\hline Typhoon type & BP model & $R^{2}$ & NARX model & $R^{2}$ \\
\hline 1 & $I_{a l w} H_{3} O_{a l}$ & 0.9977 & $I_{a l w} H_{2} O_{a l}$ & 0.9970 \\
\hline 2 & $I_{a l} H_{3} O_{a l}$ & 0.9836 & $I_{a l r} H_{4} O_{a l}$ & 0.9854 \\
\hline 3 & $I_{a l m} H_{5} O_{a l}$ & 0.9976 & $I_{a l} H_{3} O_{a l}$ & 0.9935 \\
\hline 4 & $I_{a l w m} H_{7} O_{a l}$ & 0.9974 & $I_{a l r m} H_{3} O_{a l}$ & 0.9964 \\
\hline 5 & $I_{a l m} H_{4} O_{a l}$ & 0.9953 & $I_{a l m} H_{4} O_{a l}$ & 0.9953 \\
\hline 6 & $I_{a l w} H_{6} O_{a l}$ & 0.9822 & $I_{a l r w m} H_{3} O_{a l}$ & 0.9857 \\
\hline 7 & $I_{a l m} H_{5} O_{a l}$ & 0.9834 & $I_{a l r w m} H_{3} O_{a l}$ & 0.9853 \\
\hline 8 & $I_{a l} H_{4} O_{a l}$ & 0.9902 & $I_{a l m} H_{2} O_{a l}$ & 0.9903 \\
\hline 9 & $I_{a l r w} H_{4} O_{a l}$ & 0.9955 & $I_{a l r} H_{4} O_{a l}$ & 0.9967 \\
\hline 10 & $I_{a l r w m} H_{5} O_{a l}$ & 0.9520 & $I_{a l r w} H_{3} O_{a l}$ & 0.9836 \\
\hline Average & - & 0.9875 & - & 0.9909 \\
\hline
\end{tabular}




\subsection{Forecasting of Typhoon Moving Track}

In this study, a total of 100 historical typhoon cases are considered to model the typhoon path forecasting problem, where 2 to 19 sets of typhoon data are taken for each of the ten typhoon type, according to the distribution of typhoon occurrences shown in previously section. For each typhoon type, there exists a variation of typhoon path from different cases, the mixed data set from all cases in each typhoon type may be used to develop a neural network model, but it may become too tedious and overtraining, particularly for the more often occurrence typhoon types. Instead, the present study tried to develop neural network models from each case in each type, and the relatively better models based on the performance are determined to represent the model to be used for future forecasting in each type.

Now by taking the five input parameters including longitude, latitude, cyclonic radius, wind speed at central point, and moving speed of each typhoon case, a relatively better neural network model $\left(\mathrm{I}_{5} \mathrm{H}_{n} \mathrm{O}_{2}\right.$, where $\mathrm{n}$ denotes number of neurons) based on the best performance in testing calculation stage is obtained for each typhoon type. Table 4 shows the relatively better neural network model for the ten typhoon types. It can be found that the static model in general requires more neurons in the hidden layer than that of dynamic model. Note that the special case of typhoon Type 10 does not have a regular moving track, the model developed here is for reference only, and no further analysis is performed for this unstable typhoon type. From the above discussed results, the dynamic neural network model seems more favourable to deal with this type of typhoon track forecasting problem studied herein, and so this model is taken for further analysis as follows.

By employing the dynamic neural network model for the nine typhoon types, displayed in Figure 4 is the approaching tendency of output results with target records, for all data sets in the calculation stages. It can be seen that typhoon Type 3 has the best performance, whereas typhoon Type 8 has the poorest performance. However, all models tend to have a suitable reliability for forecasting typhoon moving track from evaluation index of correlation coefficient. Figure 5 shows the convergent tendency of mean square errors (take Type 2 and Type 4 as examples), it converges to a required tolerance without too many epochs for the problems studied herein.

Table 4. The relatively better static and dynamic models for each of the ten typhoon types.

\begin{tabular}{|c|c|c|c|c|c|}
\hline Typhoon type & \multicolumn{2}{|c|}{$\mathrm{NN}$ model } & Typhoon type & \multicolumn{2}{c|}{$\mathrm{NN}$ model } \\
\hline \multirow{3}{*}{1} & $\mathrm{BP}$ & $\mathrm{I}_{5} \mathrm{H}_{4} \mathrm{O}_{2}$ & \multirow{2}{*}{6} & $\mathrm{BP}$ & $\mathrm{I}_{5} \mathrm{H}_{4} \mathrm{O}_{2}$ \\
\cline { 2 - 3 } & $\mathrm{NARX}$ & $\mathrm{I}_{5} \mathrm{H}_{2} \mathrm{O}_{2}$ & & $\mathrm{NARX}$ & $\mathrm{I}_{5} \mathrm{H}_{5} \mathrm{O}_{2}$ \\
\hline \multirow{2}{*}{2} & $\mathrm{BP}$ & $\mathrm{I}_{5} \mathrm{H}_{6} \mathrm{O}_{2}$ & \multirow{2}{*}{7} & $\mathrm{BP}$ & $\mathrm{I}_{5} \mathrm{H}_{7} \mathrm{O}_{2}$ \\
\cline { 2 - 3 } & $\mathrm{NARX}$ & $\mathrm{I}_{5} \mathrm{H}_{4} \mathrm{O}_{2}$ & & $\mathrm{NARX}$ & $\mathrm{I}_{5} \mathrm{H}_{3} \mathrm{O}_{2}$ \\
\hline \multirow{2}{*}{3} & $\mathrm{BP}$ & $\mathrm{I}_{5} \mathrm{H}_{7} \mathrm{O}_{2}$ & \multirow{2}{*}{8} & $\mathrm{BP}$ & $\mathrm{I}_{5} \mathrm{H}_{8} \mathrm{O}_{2}$ \\
\cline { 2 - 3 } & $\mathrm{NARX}$ & $\mathrm{I}_{5} \mathrm{H}_{3} \mathrm{O}_{2}$ & & $\mathrm{NARX}$ & $\mathrm{I}_{5} \mathrm{H}_{4} \mathrm{O}_{2}$ \\
\hline \multirow{3}{*}{4} & $\mathrm{BP}$ & $\mathrm{I}_{5} \mathrm{H}_{4} \mathrm{O}_{2}$ & \multirow{2}{*}{9} & $\mathrm{BP}$ & $\mathrm{I}_{5} \mathrm{H}_{7} \mathrm{O}_{2}$ \\
\cline { 2 - 3 } & $\mathrm{NARX}$ & $\mathrm{I}_{5} \mathrm{H}_{3} \mathrm{O}_{2}$ & & $\mathrm{NARX}$ & $\mathrm{I}_{5} \mathrm{H}_{4} \mathrm{O}_{2}$ \\
\hline \multirow{2}{*}{5} & $\mathrm{BP}$ & $\mathrm{I}_{5} \mathrm{H}_{8} \mathrm{O}_{2}$ & \multirow{2}{*}{10} & $\mathrm{BP}$ & $\mathrm{I}_{5} \mathrm{H}_{7} \mathrm{O}_{2}$ \\
\cline { 2 - 3 } & $\mathrm{NARX}$ & $\mathrm{I}_{5} \mathrm{H}_{3} \mathrm{O}_{2}$ & & $\mathrm{NARX}$ & $\mathrm{I}_{5} \mathrm{H}_{3} \mathrm{O}_{2}$ \\
\hline
\end{tabular}


International Journal of Artificial Intelligence and Applications (IJAIA), Vol.8, No.6, November 2017
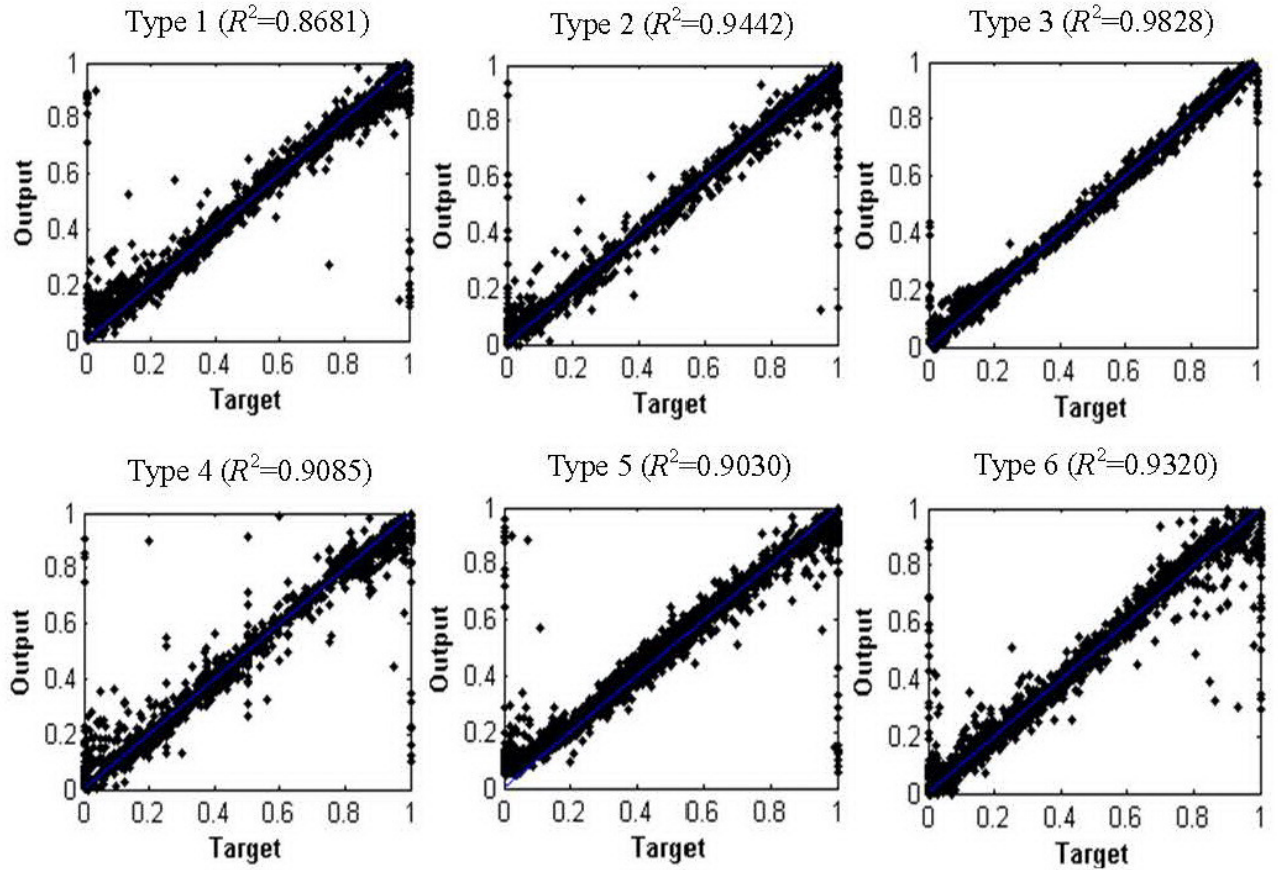

Type $5\left(R^{2}=0.9030\right)$

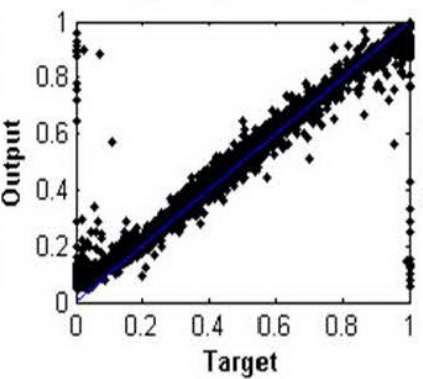

Type $6\left(R^{2}=0.9320\right)$

Type $7\left(R^{2}=0.8778\right)$

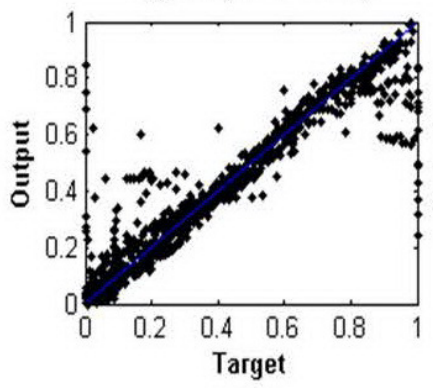

Type $8\left(R^{2}=0.7970\right)$

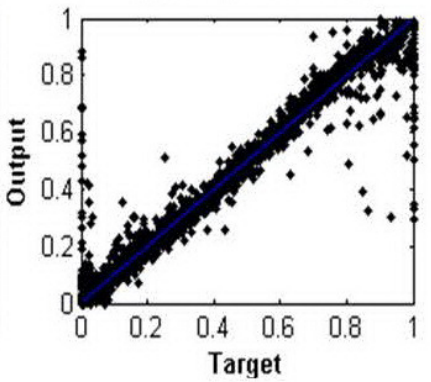

Type $9\left(R^{2}=0.9249\right)$
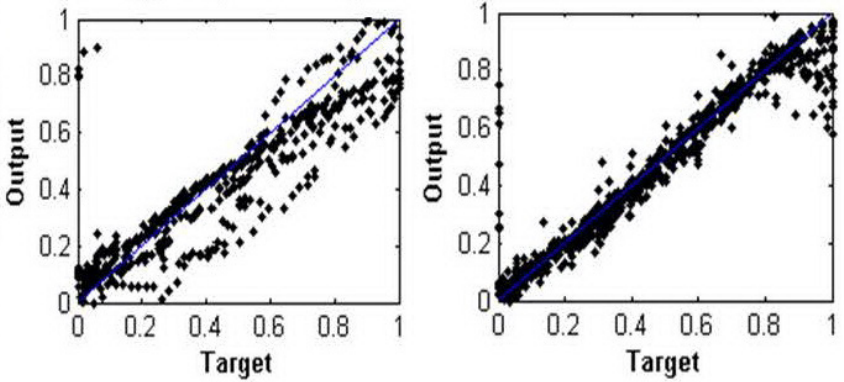

Figure 4. The approaching tendency of output values with target records.
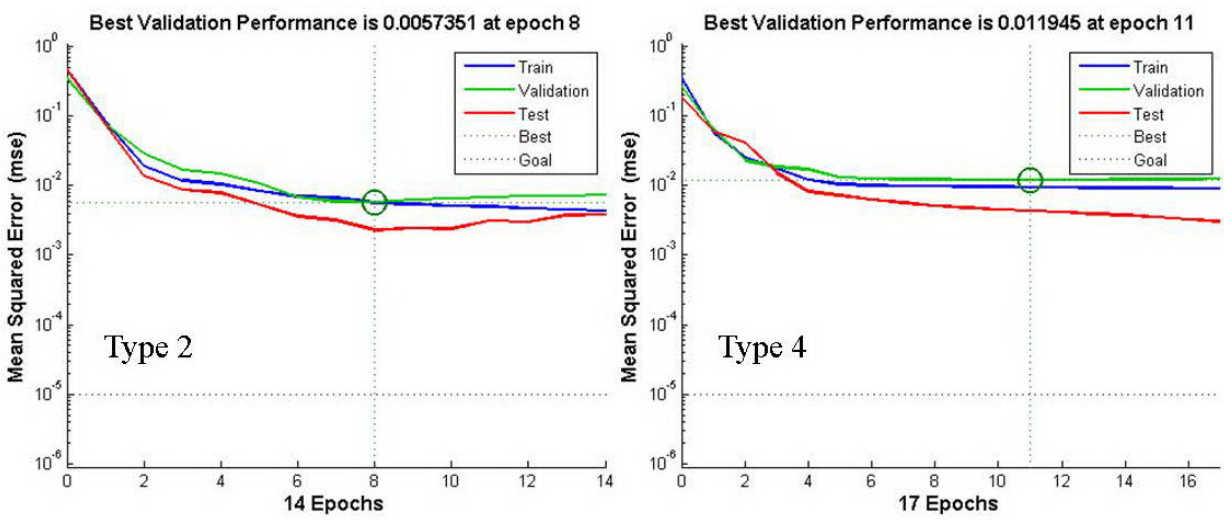

Figure 5. Convergent tendency of mean square errors (e.g. typhoons Type 2 and Type 4).

By inverting the normalized calculation results to the coordinates of typhoon central location, the forecasting typhoon track for the typhoon types with higher occurrence rates (e.g. Types 2,3,5, 
and 6) is shown in Figure 6. In general, it can be seen that the forecasting result is in good agreement with actual typhoon record. However, if a blow up of the track plots, for examples, typhoon Types 3 and Type 6 as shown in the left hand side of Figure 7, there exist some unexpected inaccurate calculation results in the middle part and the end part of the typhoon path. As one of disadvantages of using neural network approach is the possibility of converging to a local minimum during searching solution process, and that may cause the none-reasonable result as presented here in these typhoon forecasting cases.
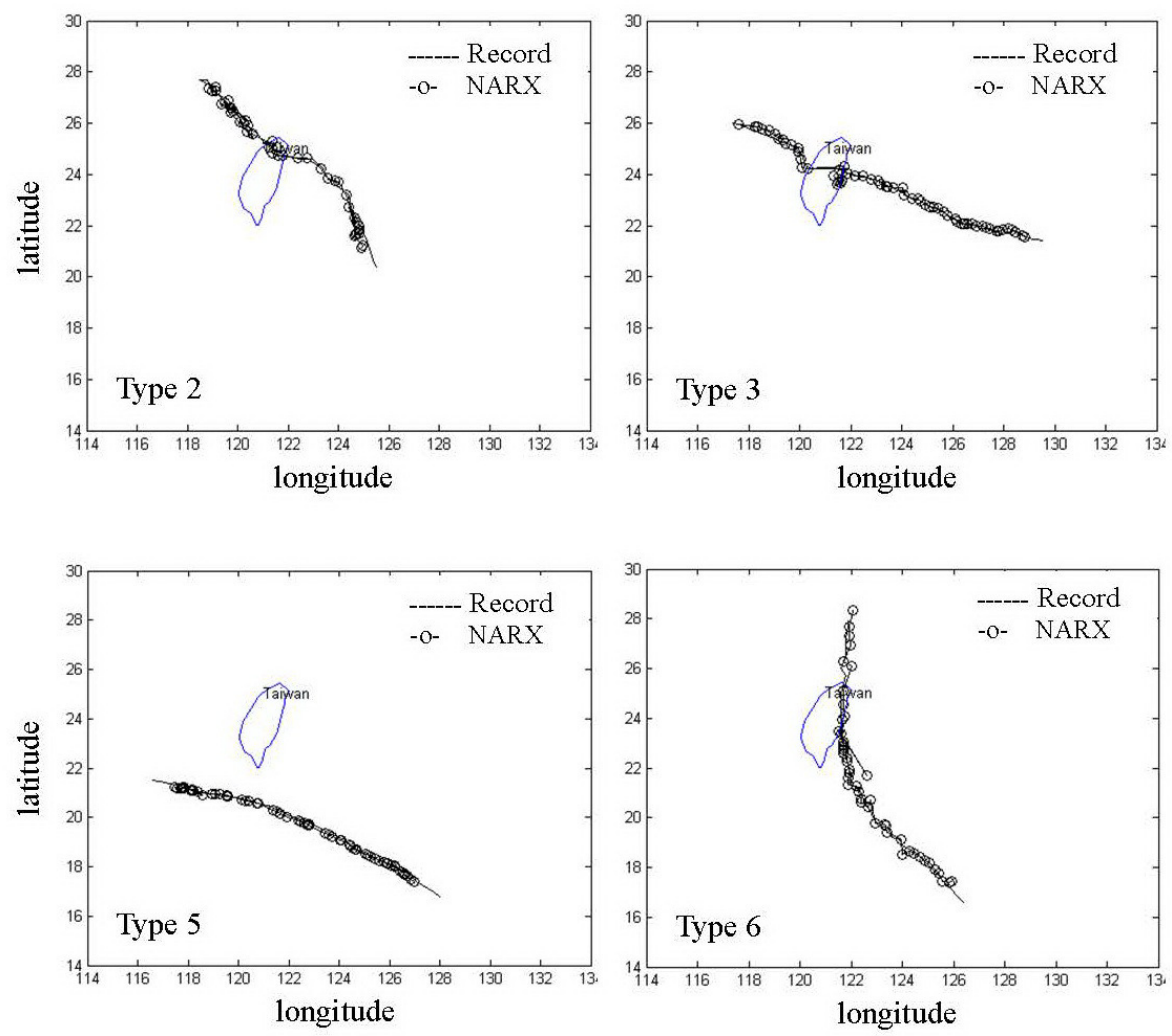

Figure 6. Forecasting results by a dynamic model compared with the actual typhoon record.

In order to improve the neural network forecasting result in the time series problems, a simple method, the so-called moving average method can be adopted, and the equation is:

$$
M a=\frac{y(t-2)+y(t-1)+y(t)}{3}
$$

where $M a$ is the correction of forecasting result; $y(t)$ is the output at time step $t$; and $n$ denotes the delay number here in this studied problem. As seen the right hand side of Figure 7, a more reasonable and smoother calculation result is obtained, which can further increase a reliability of the developed dynamic neural network model, and can be taken for future typhoon track forecasting work. 
Type 3 (original)

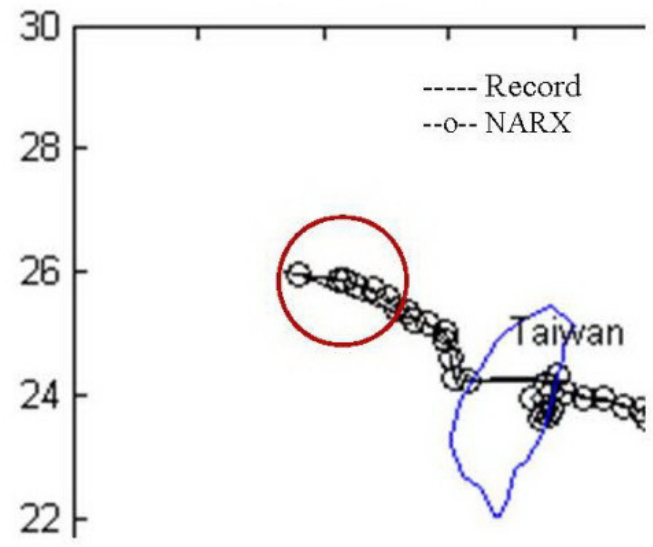

Type 6 (original)

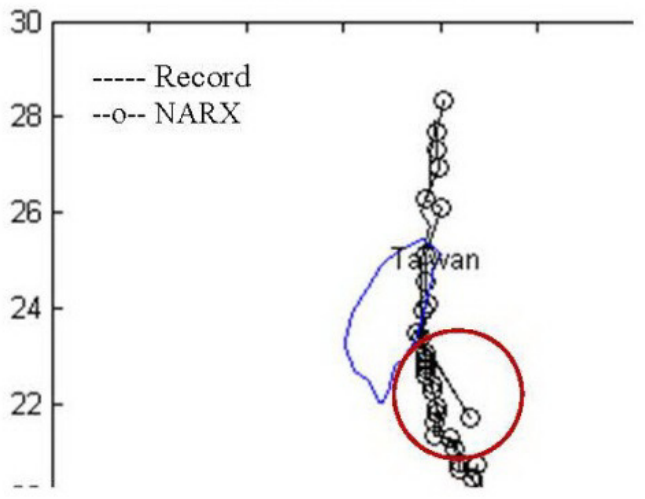

Type 3 (revised)

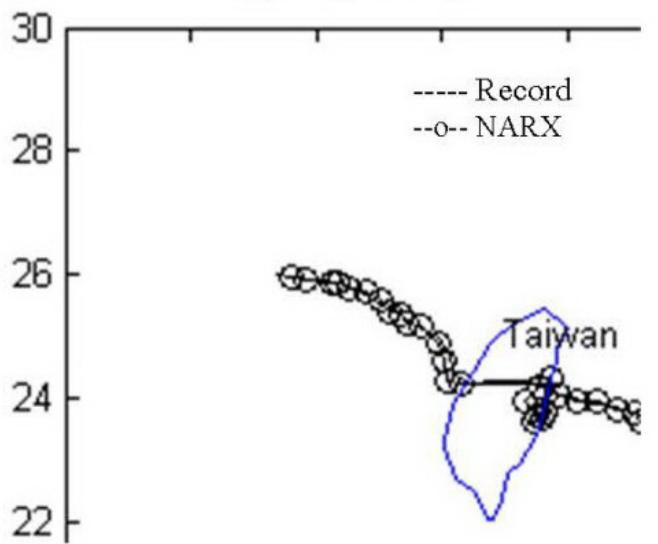

Type 6 (revised)

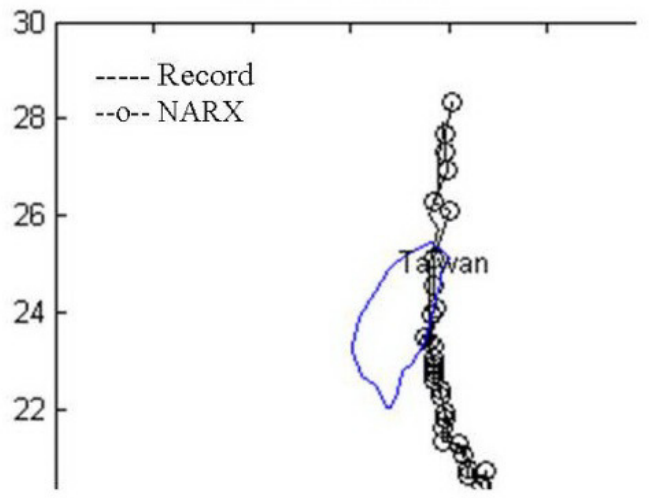

Figure 7. Blow up of forecasting result and revised result (e.g. Type 3 and Type 6).

For validating the present developed dynamic neural network model with moving average method, two more recently typhoon events, namely Typhoon Kongrey and Typhoon Nepartak occurred in 2013 and 2016, respectively are taken for analysis. At the beginning, the first typhoon is judged as Type 6, whereas the second typhoon belongs to Type 4, based on the potential moving track of the two typhoons. Then, by applying the relatively better neural network models, i.e. by using the weight and bias values in the model developed previously without resort to any further training. The directly forecasting result is shown in Figure 8, and acceptable typhoon track forecasting results are found for both typhoons.

\section{CONCLUSIONS}

Typhoon is one of natural disasters in the earth, and that may bring a huge amount of rainfall to cause property damages and loss of human lives. To track the movement of a typhoon, which may be taken to evaluate the negative effects in the neighborhood areas, various statistical and numerical models have been reported previously. However, these complicated models in 

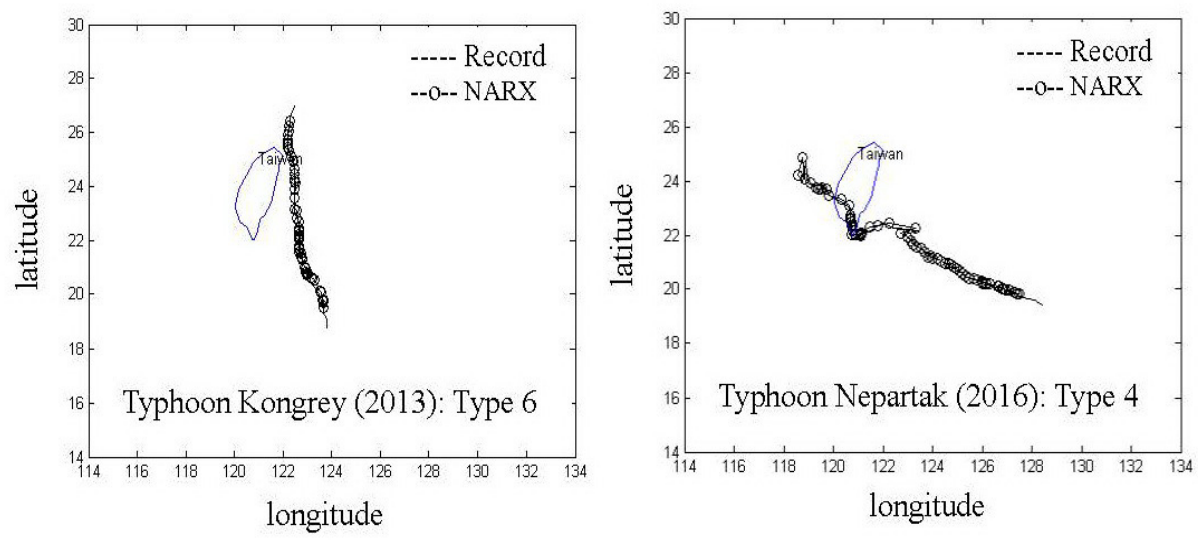

Figure 8. Directly forecasting results by a dynamic model with moving average method.

general require many weather information and computer resources, and those may cost expensive. To facilitate the typhoon path forecasting work, this study proposed a method of using both static and dynamic neural network models to solve the problem effectively in Taiwan region.

The key elements for developing the model include typhoon central location (longitude, latitude), cyclonic radius, central wind speed, and moving speed. By varying different neurons in the hidden layer, a relatively better neural network model is obtained for each of the ten types of typhoon classified in the island of Taiwan. Both static and dynamic models exhibited a good performance, but the dynamic model seems to have a slightly better evaluation index than that of the static model. In particularly, this nonlinear autoregressive network with the use of moving average method, could improve some of the inadequate forecasting result that may generate from a local minimum converged by the neural network approach.

Due to the limitation of typhoon data resources, the neural network model developed here in this study did not consider typhoon parameters at the early stage, and so the model could not be applied to forecast the typhoon moving track at its early stage. Once the typhoon data can be obtained more in detail, the present neural network approach may help to forecast a typhoon potential track, and that may help to provide a warning message as soon as possible. For future study, some of other typhoon related information such as atmospheric pressure, sea temperature, wind direction, topographic condition, and season factor, may be taken into account to model the problem for increasing its reliability. Besides, a global searching capability of genetic algorithm may be incorporated into neural computing to prevent the convergence of local minimum. Nonetheless, the present study did show a simple way to deal with this type of typhoon track forecasting problem, and may be extended to increase its applicability.

\section{ACKNOWLEDGEMENTS}

The authors would like to thank the Central weather Bureau of Taiwan for providing historical typhoon records.

\section{REFERENCES}

[1] Central Weather Bureau, (2016), "Knowledge of typhoons, one hundred questions of typhoons," Ministry of Transportation, Taiwan, http://www.cwb.gov.tw/V7/knowledge/encyclopedia/ ty000.htm.

[2] Fett, R.W. and Brand, S., (1975), "Tropical cyclone movement forecasts based on observations from satellites," Journal of Applied Meteorology, 14(4): 452-465. 
[3] Marshall, J.F.L., Leslie, L.M., Abbey Jr. R.F. and Qi, L., (2002), “Tropical cyclone track and intensity prediction: The generation and assimilation of high-density, satellite-derived data," Meteorology and Atmospheric Physics, 80(1-4): 43-57.

[4] National Hurricane Center, (2009), "Technical summary of the national hurricane center track and intensity models," National Oceanic and Atmospheric Administration. pp. 1-18.

[5] Roy, C. and Kovordanyi, R., (2012) "Tropical cyclone track forecasting techniques: a review," Atmospheric Research, 104-105: 40-69.

[6] Rozanova, O.S., Yu, J.L. and Hu, C.K., (2010), "Typhoon eye trajectory based on a mathematical model: Comparing with observational data," Nonlinear Analysis: Real World Applications 11: 1847 1861.

[7] Hsiao, L.F., Peng, M.S., Chen, D.S. and Huang, K.N., (2009), "Sensitivity of typhoon track predictions in a regional prediction system to initial and lateral boundary conditions," Journal of Applied Meteorology and Climatology, American Meteorological Society, 48: 1913-1928.

[8] Van Thu, T. and Krishnamurti, T.N., (1992), "Vortex initialization for typhoon track prediction," Meteorology and Atmospheric Physics, 47(2): 117-126.

[9] Qian, W., Shan, X., Liang, H., Huang, J., Leung, C.H. and Yeh, T.C., (2014), “A generalized betaadvection model to improve unusual typhoon track prediction by decomposing total flow into climatic and anomalous flows," Journal of Geophysical Research, 119(3): 1097-1117.

[10] Lo, D.C., Wei, C.C. and Tsai, E.P., (2015), "Parameter automatic calibration approach for neuralnetwork-based cyclonic precipitation forecast models," Water, 7: 3963-3977.

[11] Chang, H.K. and Chien, W.A., (2006), "Neural network with multi-trend simulating transfer function for forecasting typhoon wave," Advances in Engineering Software, 37: 184-194.

[12] Lee, T.L., (2008), "Prediction of storm surge and surge deviation using a neural network," Journal of Coastal Research, 24(4A): 76-82.

[13] Huang, X.Y., Jin, L. and Shi, X.M., (2011), “A nonlinear artificial intelligence ensemble prediction model based on EOF for typhoon track," Fifth International Joint Conference on Computational Sciences and Optimization, pp. 1329-1333.

[14] Chang, B.R. and Tsai, H.F., (2007), "Forecasting typhoon moving path using BPNN-weighted greyC3LSP tuned by quantum-neuron-based adaptive support vector regression with nested local adiabatic evolution," Intelligent Information Hiding and Multimedia Signal Processing, 1: 3-6.

[15] Central Weather Bureau, (2016), "Typhoon data base," Ministry of Transportation, Taiwan, http://rdc28.cwb.gov.tw/TDB/ntdb/pageControl/basic.

[16] Hornik, K., Stinchcombe, M. and White, H., (1989), "Multilayer feedforward networks are universal approximators," Neural Networks, 2(5): 359-366.

[17] Kamali, R. Binesh, A.R., (2013), "A comparison of neural networks and adaptive neuro-fuzzy inference systems for the prediction of water diffusion through carbon nanotubes, Microfluidics and Nanofluidics, 14(3): 575-581.

[18] Mukerji, A., Chatterjee, C. and Raghuwanshi, N.S., (2009), "Flood forecasting using ann, neuro-fuzzy, and neuro-ga models. Journal of Hydrologic Engineering, 14(6): 647-652.

[19] Mert, I., Karakuş, C. and Üneş, F., (2016), "Estimating the energy production of the wind turbine using artificial neural network, Neural Computing and Applications, 27(5): 1231-1244.

[20] Murthy, V.M.S.R., Kumar, A. and Sinha, P.K., (2016), "Prediction of throw in bench blasting using neural networks: an approach," Neural Computing and Applications, 1-14.

[21] Abounoori, A.A., Mohammadali, H., Alikhani, N.G. and Naderi, E., (2012), "Comparative study of static and dynamic neural network models for nonlinear time series forecasting," MPRA Paper No. 46466, 1-18.

[22] Schmidhuber,J., (1989), "A local learning algorithm for dynamic feedforward and recurrent networks," Connection Science, 1(4): 403-412.

[23] Trischler, A.P. and D’Eleuterio, G.M.T., (2016), "Synthesis of recurrent neural networks for dynamical system simulation," Neural Networks, 80: 67-78.

[24] Arriandiaga, A., Portillo, E., Sánchez, J. A., Cabanes, I. and Pombo, I., (2016), "A new approach for dynamic modelling of energy consumption in the grinding process using recurrent neural networks," Neural Computing and Applications, 27(6): 1577-1592.

[25] El-Shafie, A., Noureldin, A., Taha, M., Hussain, A. and Mukhlisin, M., (2012), "Dynamic versus static neural network model for rainfall forecasting at Klang river basin, Malaysia," Hydrology and Earth System Sciences, 16: 1151-1169.

[26] Chang, F.J. and Chang, L.C., (2010), "Introduction of artificial neural network - principles and applications," Tsanghai Publishing, Taiwan. 
International Journal of Artificial Intelligence and Applications (IJAIA), Vol.8, No.6, November 2017

[27] Chang, D.F., (2009), "Neural network applied design by MATLAB," China Machine Press, China.

[28] Demuth, D. and Beale, M., (2002), "Neural network toolbox user guide," online, The Mathworks, Inc., USA.

[29] Haykin, S.S., (1999), Neural networks - a comprehensive foundation, Prentice Hall, USA.

[30] Fu, L.M., (1994), "Neural networks in computer intelligence,” McGraw-Hill, Inc., USA.

[31] Williams, R.J. and Zipser, D., (1994), "Gradient-based learning algorithms for recurrent networks and their computational complexity," Backpropagation: Theory, Architectures, and Applications, Eds. by Y. Chauvin and D.E. Rumelhart, Lawrence Erlbaum Associates, Hillsdale, NJ, 433-486.

[32] Williams R.J. and Peng J., (1990), "An efficient gradient-based algorithm for on line training of recurrent network trajectories", Neural Computation 2: 49

\section{Authors}

Tienfuan Kerh received his Ph.D degree in Civil Engineering from University of Southern California, USA. Currently, he is a full professor in civil engineering department, National Pingtung University of Science and Technology, Taiwan. He ever served as a visiting scholar in University of Sydney, Australia; University of London (University College London \& Imperial College London), U.K.; University of Heidelberg, Germany; and University of California, Irvine, USA. His scientific interests include artificial intelligence applications in seismic data analysis, coastal line variation, and water resources. He also works in the field of computational fluid dynamics,

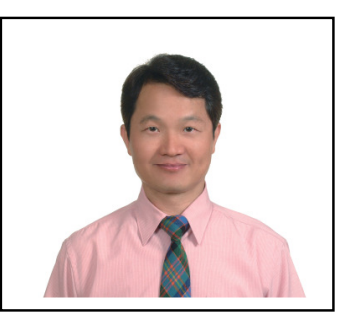
emphasized in fluid-structure interaction. In addition to publish numerous journal and conference papers, he serves as an editorial member for several international journals and conferences. He also served as a reviewer for more than 20 international journals as listed in Science Citation Index. 